\title{
Red light-emitting electrochemical cells employing pyridazine- bridged cationic diiridium complexes
}

\author{
Maria-Grazia La-Placa, ${ }^{a}$ Ana María Igual-Muñoz, ${ }^{a}$ Jorge Romero, ${ }^{\text {a }}$ Ruth E.

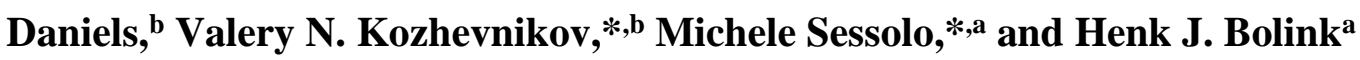 \\ a Instituto de Ciencia Molecular (ICMol), Universidad de Valencia, Catedrático José Beltrán, 2, 46980 \\ Paterna, Spain. E-mail: michele.sessolo@uv.es \\ ${ }^{\mathrm{b}}$ Department of Applied Sciences, Northumbria University, Newcastle upon Tyne, NE1 8ST, UK. E-mail: \\ valery.kozhevnikov@northumbria.ac.uk.
}

\begin{abstract}
A rigid dinuclear Ir(III) complex showing high photoluminescence quantum yield in pure films was successfully used to fabricate light-emitting electrochemical cells with and without ionic liquid additives. The devices showed nearly instantaneous electroluminescence after biasing and maximum quantum yield approaching $1 \%$. The lifetime of the devices was found to be limited to approximately 20 hours, which we correlated with the irreversible oxidation of the complex as seen from electrochemical measurements. This work validates the use of highly luminescent dinuclear iridium complexes in light-emitting electrochemical cells. Future studies will pursue materials with more efficient photoluminescence as well as improved electrochemical stability.
\end{abstract}

Light-emitting electrochemical cells (LECs) are relatively simple electroluminescent device that can be fabricated from solution on large area and therefore would be the most cost effective technology for lighting applications.[1,2] The main feature of LECs is the inclusion of ionic species in an organic semiconductors, capable of assisting charge injection, transport and recombination within a single active layer.[3-5] Despite extensive research effort, LECs are less efficient and stable than organic light-emitting diodes, and the search for new materials and device architectures is ongoing. The most efficient LECs are based on phosphorescent emitters, in particular cyclometallated $\operatorname{Ir}(\mathrm{III})$ complexes that are able to convert all bound electron-hole pairs to emissive triplet excitons.[6-8] A large 
variety of monoiridium complexes has been evaluated in LECs. Dinuclear and polynuclers compounds, containing two or more metal centres in one luminophore, offer additional degrees of freedom in the design of new phosphorescent materials, i.e. i) the choice of the bridging ligand modulating flexibility, ii) increase of spin-orbit coupling as a consequence of multiple metal centres, iii) possible stabilization of the complex due to chelating properties of the bridging ligand.[9] However polynuclear Ir(III) compounds have been scarcely investigated in LECs.[9] In a recent work, the structure and photophysical properties of two tetranuclear complexes based on $\operatorname{Ir}_{2} \mathrm{M}_{2}$ clusters $(\mathrm{M}=\mathrm{Ag}$ or $\mathrm{Cu}$ ), together with their diiridium precursor which is stabilized by a double alkynyl bridging system, were presented.[10] While electroluminescence from the tetranuclear complexes was indeed observed, LECs based on the dinuclear iridium compound were not fabricated. More recently, a highly luminescent pyrazolate-bridged platinum(II) complex was synthesized and used as the active layer in LECs, obtaining moderate luminescence intensity and lifetime.[11] To the best of our knowledge, however, up to now there is only one example on the application of diiridium complexes in LECs.[12] However, an oligo(phenyleneethynylene) ligand was used to bridge the two ionic complexes, resulting in a low energy excited state with significant triplet ligand-centred $\left({ }^{3} \mathrm{LC}\right)$ character and no contribution from the $\operatorname{Ir}(\mathrm{III})$ centres.[13] This excited state inevitably acts as an exciton trap, leading to low PLQY and hence weak electroluminescence intensity $\left(<4 \mathrm{~cd} / \mathrm{m}^{2}\right)$. One distinct family of highly luminescent multinuclear complexes has recently emerged. In these materials, rigid ditopic ligands are employed and the metallic centres are bridged by heterocycle such as pyrimidine, pyrazine or pyridazine. By bridging metal centres through coordination to the same heterocycle (i.e. pyrimidine) as well as sharing a second ligand any rotation is hindered resulting in conformationally rigid molecules. In these systems, the introduction of additional metal centres leads to significant red-shifts compared to monometallic analogues, which, importantly, do not come at the expense of efficiency. This feature has been found to arise from substantial increases in radiative decay rate, leading to high quantum efficiencies and shortened emission lifetimes, both of which are desirable for electroluminescence.[14, 15] Moreover, due to the conformational rigidity imparted by the coordination of additional metal ions, non-radiative decay is minimized, and the complexes show narrower emission profiles that can favour high colour purity. Following this design, diiridium complexes with PLQY approaching unity have been recently demonstrated.[14, 16] Importantly, these complexes show very high values of radiative 
decay and short lifetime of emission, characteristics that may minimise triplet-triplet annihilation within the emissive layer of LECs. Motivated by these properties we decided to evaluate this class of emitters in LECs. In this work, we report the first example of LECs based on rigidly-linked dinuclear Ir(III) complex as the electroluminescent material.

\section{Materials and Methods}

Compound characterization - The cationic cyclometallated di-iridium (II) complex-1 (Fig. 1a) was synthesized following previously reported protocols.[17] The samples for thin-film photoluminescence (PL) measurements were prepared as pure complex and also with the same composition and thickness as the emissive layer used in LECs. For this, the complex was mixed with the ionic liquid (IL) 1-butyl-3-methylimidazolium hexafluorophosphate $\left([\mathrm{Bmim}]\left[\mathrm{PF}_{6}\right]\right)$ in a 4-to-1 molar ratio. A $100 \mathrm{~nm}$ thick film was deposited from a $20 \mathrm{mg} \mathrm{mL}^{-1}$ solution of complexes-1 in acetonitrile. Prior to deposition, all solutions were filtered with a $0.22 \mu \mathrm{m}$ pore size filter and spin-coated at $1000 \mathrm{rpm}$ for $30 \mathrm{~s}$ in air onto cleaned quartz substrates. Quantum yields and PL spectra in thin films were recorded using a Hamamatsu absolute quantum yield C9920 spectrometer. PL decays were measured using a compact fluorescence lifetime spectrometer C11367, Quantaurus-Tau. PL lifetime measurement software U11487 was used to register the data; the PL decays were fitted with a biexponential function. UV-Vis absorption spectra were recorded with an Avantes fibre optics spectrometer. Ambient Pressure Photoemission Spectroscopy (APS) were measured using an APS02 system by KP Technology. The setup includes a dual-mode APS unit to measure the absolute work function of a material with an excitation range of $3.4 \mathrm{eV}$ to $7.0 \mathrm{eV}$. The absolute ionization potential was estimated by extrapolation of the cube root of the photoemission.

Device fabrication - All materials were used as received. Poly(3,4ethylenedioxythiophene):polystyrene sulfonate (PEDOT:PSS, Clevios P VP Al 4083) was purchased from Heraeus. $[\mathrm{Bmim}]\left[\mathrm{PF}_{6}\right]$ and acetonitrile were purchased from Sigma Aldrich. The photolithography-patterned indium tin oxide (ITO) glass substrates were purchased from Naranjo Substrates (www.naranjosubstrates.com). The substrates were subsequently cleaned with soap, deionized water, and isopropanol in an ultrasonic bath for 5 minutes each, followed by 20 minutes UV-ozone treatment. Onto the clean ITO 
substrates, an $80 \mathrm{~nm}$ thick film of PEDOT:PSS was spin-coated at $1000 \mathrm{rpm}$ for 1 minute. The PEDOT:PSS dispersion was filtered with a $0.45 \mu \mathrm{m}$ pore size filter. The layers were dried at $150{ }^{\circ} \mathrm{C}$ for 15 minutes. On top of it, a $100 \mathrm{~nm}$ thick film of the emissive layer was deposited in the same conditions described above for PL measurements. After deposition, they were transferred to a nitrogen glove box and annealed at $100{ }^{\circ} \mathrm{C}$ for 1 hour. Finally, a $70 \mathrm{~nm}$ thick aluminium film was thermally deposited in vacuum as the top electrode using a shadow mask.

LECs characterization - LECs were tested by applying pulsed current and by monitoring the voltage and the luminance with a True Colour Sensor (MTCSiCT Sensor, MAZeT GimbH) using a Lifetime Test System (Botest OLT OLED Lifetime-Test System, Botest System $\mathrm{GmbH}$ ). The pulsed current consisted of a block wave at $1000 \mathrm{~Hz}$ frequency with a $50 \%$ duty cycle. The current density at the peak of the pulse was $200 \mathrm{~A} \mathrm{~m}^{-2}$ and the average current density was $100 \mathrm{~A} \mathrm{~m}^{-2}$. Electroluminescence spectra were recorded using an Avantes fibre optics photo-spectrometer. All devices were tested without encapsulation inside a nitrogen glove-box at room temperature.

Electrochemical measurements - The electrochemical experiments were performed using a Gamry electrochemical workstation (Gamry 1000E potentiostat/galvanostat) connected to a personal computer that uses Gamry global electrochemical software. The powdered materials $(2 \mathrm{mg})$ were mixed in $2 \mathrm{~mL}$ of Nafion and ethanol (1:1) and deposited on a 3 mm diameter glassy carbon disc working electrode, which was polished sequentially with 0.3, 0.1 and $0.05 \mu \mathrm{m}$ alumina powders and washed with deionised water before each experiment. A typical three-electrode experimental cell equipped with a platinum wire as the counter electrode and a silver wire as the pseudoreference electrode was used for the electrochemical characterization of the working electrodes. All measurements were carried out with nitrogen bubbling. The electrochemical properties were studied measuring the $\mathrm{CV}$ at a scan rate of $100 \mathrm{mV} \cdot \mathrm{s}^{-1}$ in $0.1 \mathrm{M} \mathrm{TBAPF} / \mathrm{CH}_{3} \mathrm{CN}$ solution. Ferrocene was added as an internal standard upon completion of each experiment. All potentials are reported in $\mathrm{V}$ versus $\mathrm{Ag} / \mathrm{AgCl}$.

\section{Results and discussion}

The compound chosen for this study is a cationic cyclometallated di-iridium (II) complex-1 which we previously developed for bioimaging applications (Fig. 1a).[17] 
The bridging pyridazine ligand brings the two cyclometallated $\operatorname{Ir}(\mathrm{III})$ metal centres close together so that the single chloride atom also acts as the second bridging ligand, leading to a cationic complex. Because symmetric terdentate NCN ligands are used, the complex is achiral despite the presence of two octahedral Ir centres. The lack of chiral centres eliminate the problem of the formation of diastereomers and simplifies the synthesis. Due to ionic nature of the complex, the solubility in polar solvent such as acetonitrile is sufficiently high to obtain high quality thin films. Thus spin-coating acetonitrile solutions $(20 \mathrm{mg} / \mathrm{mL})$ at $1000 \mathrm{rpm}$ produced films of the material of about $100 \mathrm{~nm}$ thickness. The absorption spectrum of the thin film (Fig. 1b) shows a steep absorption until $500 \mathrm{~nm}$, corresponding to a bandgap of approximately $2.5 \mathrm{eV}$. We measured the thin-film photoluminescence (PL) by exciting the sample at $400 \mathrm{~nm}$ in an integrated sphere, in order to estimate the PLQY. The PL spectrum (Fig. 2b) consists of a broad asymmetric signal with maximum at 598 and full width at half maximum (FWHM) of $111 \mathrm{~nm}$. The PLQY was found to be $33 \%$, which is lower than the value reported for deaerated acetonitrile solution (62\%).[17]

a)

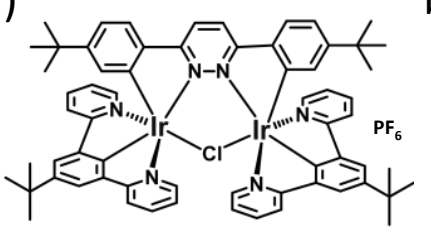

b) 0.5

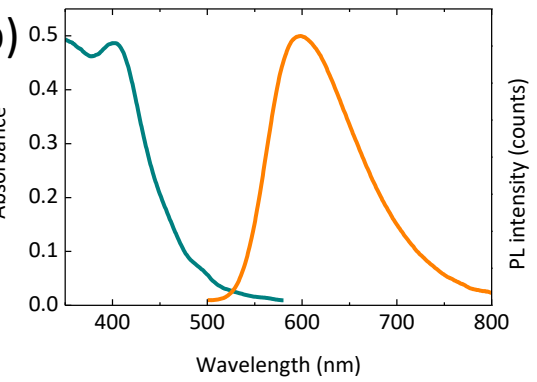

c)

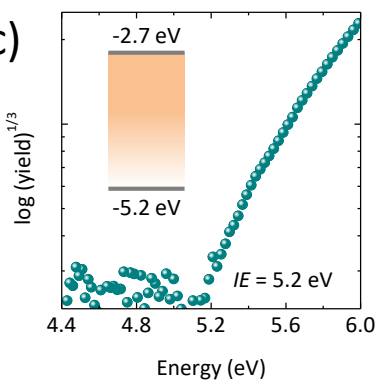

Fig. 1 (a) Chemical structure of the ionic complex 1. (b) Absorption (left) and photoluminescence (right) spectra of $100 \mathrm{~nm}$ thick film of complex 1 on quartz (excitation wavelength $400 \mathrm{~nm}$ ). (c) Photoemission spectrum of a thin film of the same compound deposited on ITO. The inset depicts an estimation of the energy levels of complex 1.

Annealing the films of complex-1 in a nitrogen glove box at $100{ }^{\circ} \mathrm{C}$ for 1 hour, did not improve PLQY. This suggests that the diminished PLQY in the solid state is not due to oxygen quenching but more likely due to a concentration quenching effect. We also characterized the ionization energy (IE) of complex-1 by means of photoemission 
spectroscopy in air (Fig. 1c). We measured an ionization potential of $5.2 \mathrm{eV}$, which can be approximated with the energy of the highest occupied molecular orbital (HOMO) of the compound. By estimating the energy gap of the material from the absorption spectrum (Fig. 1a), we obtain an electronic affinity of $2.7 \mathrm{eV}$, which allows us to draw the approximate energy levels of complex-1 (inset of Fig. 1c).

LECs were prepared on pre-patterned indium tin oxide (ITO) coated glass slides. PEDOT:PSS was used as the hole injection layer (HIL) to smooth the ITO electrode and enlarge its work function. The emissive layer was spin-coated from acetonitrile solutions of complex-1, both as a pure film (device 1) and in the presence of the ionic liquid (IL) 1-ethyl-3-methylimidazolium hexafluorophosphate $\left([\mathrm{Bmim}]\left[\mathrm{PF}_{6}\right]\right)$ in a 4:1 molar ratio (device 2). The addition of ionic liquid reduces the device turn-on time $\left(t_{\mathrm{on}}\right),{ }^{[18]}$ defined here as the time to initially reach a luminance of $100 \mathrm{~cd} \mathrm{~m}^{-2}$. LECs were finished by vacuum deposition of an aluminium cathode $(100 \mathrm{~nm})$ and were tested in a nitrogen filled glove box without encapsulation. LECs were operated applying a pulsed-current with an average current density of $100 \mathrm{~A} \mathrm{~m}^{-2}$, consisting of a block wave at $1 \mathrm{kHz}$ frequency with a duty cycle of $50 \%$.

The luminescence was continuously monitored for 120 hours (5 days, Fig. 2 ) in order to extract the characteristic device parameters, reported in Table 1. Both devices turn on immediately after biasing (inset of Fig. 2a), with an initial luminance of $38 \mathrm{~cd} / \mathrm{m}^{2}$ and 97 $\mathrm{cd} / \mathrm{m}^{2}$ for the device 1 and device 2 respectfully. $t_{o n}$ is hence very short for the device with IL (5 s) and only slightly longer (26 s) for the device with the pure complex layer. This is expected due to the larger ionic density and mobility in presence of the IL. The short turn on time observed here can be ascribed to the energy level alignment in the device. Evaporated aluminium cathodes give good electron injection in LECs, due to a local metal oxidation (and hence reduction of surface species in the active layer)[19] rather than to its work function (some limitations, however, exist, as clear from the more intense electroluminescence in the presence of IL).[20] On the other hand, the HOMO level of complex-1 lies at about $5.2 \mathrm{eV}$ from the vacuum level, approximately equal to the PEDOT:PSS work function.[21] Hence LECs based on complex-1 virtually do not suffer from injection limitations, resulting in instantaneous electroluminescence after biasing. 

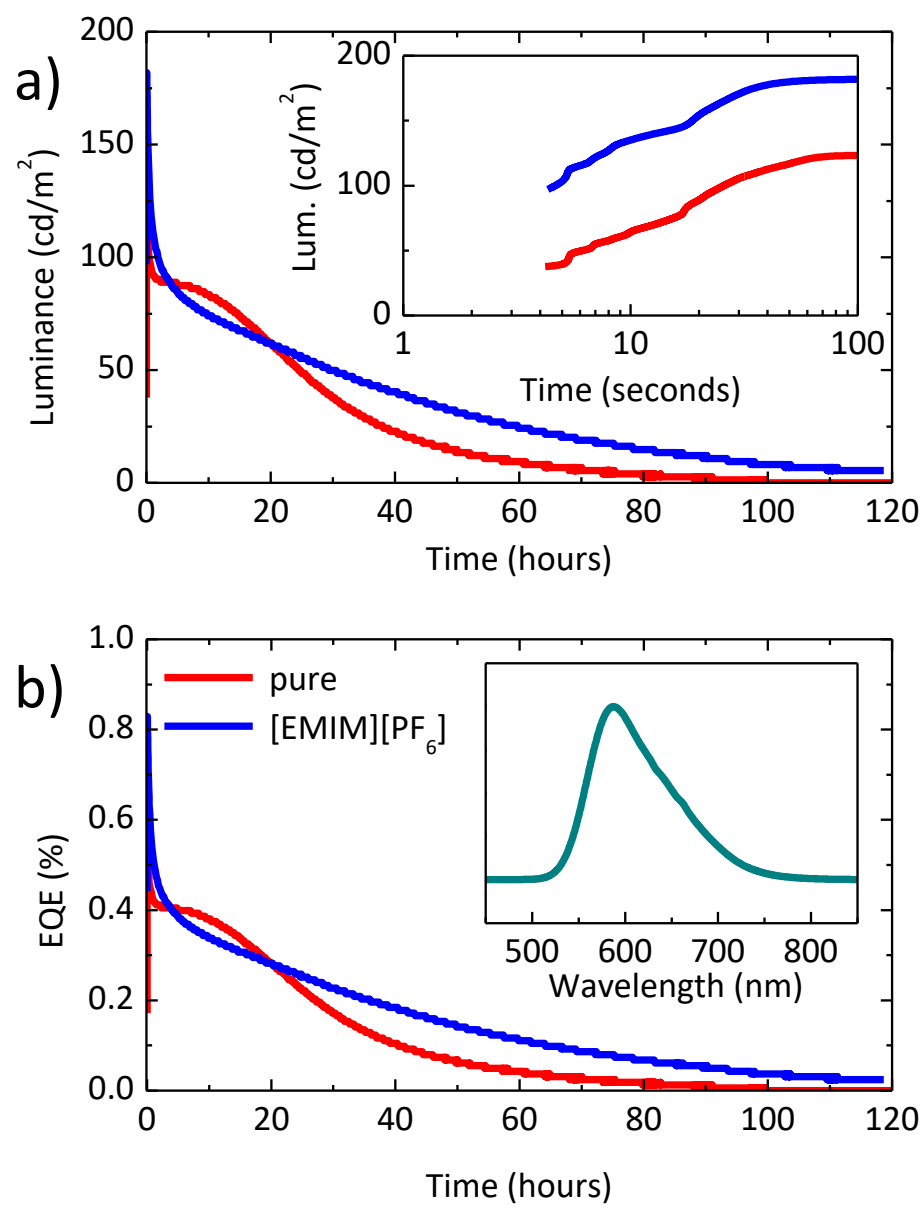

Fig. 2 Time evolution of the (a) luminance and (b) corresponding EQE for LECs employing with (blue) and without (red) ionic liquid. The inset in (a) show a zoom of the initial device operation in log scale. The inset in (b) shows the electroluminescence spectra of complex 1 .

The electroluminescence intensity rapidly increases in the first 2 minutes of operation, reaching a maximum luminance (Lum.max) of $124 \mathrm{~cd} / \mathrm{m}^{2}$ and $182 \mathrm{~cd} / \mathrm{m}^{2}$ for the devices without and with the IL, respectively. These values correspond to a maximum efficacy $(E f f \cdot \max )$ of 1.2 and $1.8 \mathrm{~cd} / \mathrm{A}$, with associated maximum external quantum efficiency (EQE $E_{\max }$, Fig. $2 \mathrm{~b}$ ) of $0.56 \%$ and $0.83 \%$. The increased efficiency for the devices in the presence of IL is not related with a difference in the PLQY, which is only slightly higher (36\%, Figure S2) compared to a pure complex-1 film (33\%). The difference is more likely related to a more balanced charge injection and transport, which is also supported by the highest initial luminance of the LECs with IL. 


\begin{tabular}{lccccc}
\hline \multicolumn{7}{c}{ Table 1 } & \multicolumn{5}{c}{ Performance indicators for LECs driven at $100 \mathrm{~A} / \mathrm{m}^{2}}$. \\
\hline Sample & $t_{\text {on }}$ & $\begin{array}{c}\text { Lum.max } \\
\left(\mathrm{cd} / \mathrm{m}^{2}\right)\end{array}$ & $\begin{array}{c}\text { Eff.max } \\
(\mathrm{cd} / \mathrm{A})\end{array}$ & $\begin{array}{c}E Q E_{\max } \\
(\%)\end{array}$ & $\begin{array}{c}t_{1 / 2} \\
(\mathrm{~h})\end{array}$ \\
\hline Device 1 & 26 & 124 & 1.2 & 0.56 & 19.7 \\
Device 2 & 5 & 182 & 1.8 & 0.83 & 3.2 \\
\hline
\end{tabular}

The electroluminescence spectra of complex-1 (inset of Fig. 2b) is slightly blue-shifted compared to its photoluminescence, with maximum at $588 \mathrm{~nm}$, and colour coordinate $(0.55,0.44)$ corresponding to the orange-yellow part of the CIE 1931 colour space (Figure S3). The device-2 with IL additives shows a monotonic luminescence decay, with a lifetime ( $t_{1 / 2}$, time to decay to half of the maximum luminance) of 3.2 hours. The luminance of the LECs with a pure active layer shows a longer $t_{1 / 2}(19.7$ hours $)$ and a more complex time-evolution, with an initial fast decay followed by a period of stabilization during the first 10 hours, after which the electroluminescence starts to decreases again. Such decay kinetics is reproducible and was observed also for devices driven with an average current density of $50 \mathrm{~A} / \mathrm{m}^{2}$ (Figure S4). These features likely indicate the occurrence of electrochemical reactions upon the injection of charge carriers from the electrodes into the complex-1 layer.

In order to shed light on the relatively low stability of the compounds in e LECs, we studied its electrochemical stability by means of cyclic voltammetry $(\mathrm{CV})$ and differential pulse voltammetry (DPV). CV and DPV show a reversible redox peak at negative potentials $(-0.6 \mathrm{~V})$, and two irreversible oxidation waves at 0.64 and $1.32 \mathrm{~V}$ (Fig. 3). These signals can also be identified in the DPV (approximately at 1.0 and $1.5 \mathrm{~V}$, respectively). The shift is likely due to the complex degradation upon measurement, as the DPV was carried out after the CV. The electrochemistry suggests that the most probable cause of degradation is the oxidation of the complex, hindering an efficient and balanced charge transport within the device. If we consider a non-ohmic hole injection, the initial exciton formation and recombination would take place in the proximity of the anode. As holes are injected, the material would start to decompose progressively towards the centre of the active layer, effectively quenching the electroluminescence. The differences observed in the decay of the pure film device-1 as compared to device-2 arise 
from a larger ionic conductivity of the latter, accelerating the formation of doped zones and probably masking the slow electrochemical reaction happening at the anode side.

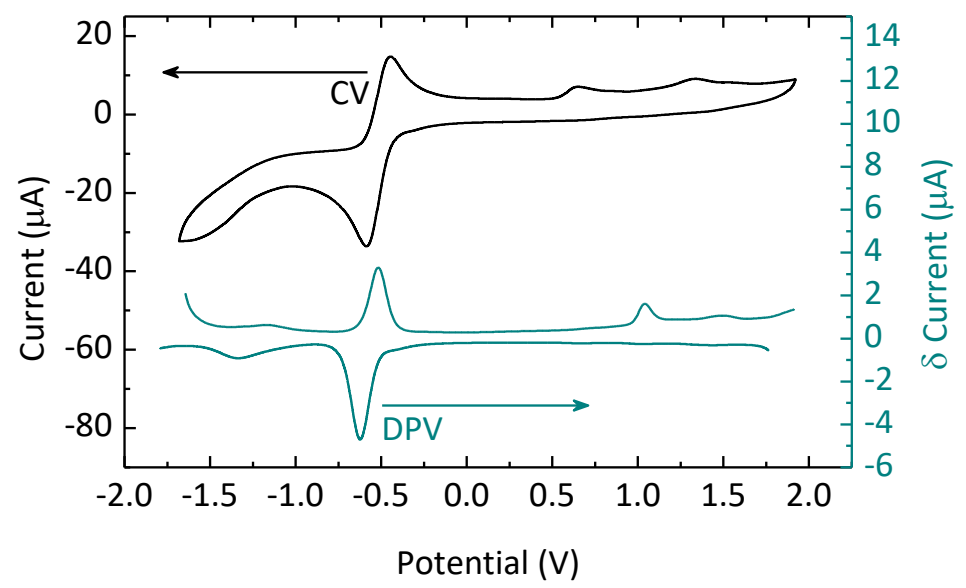

Fig. 3 Solid-state Cyclic Voltammetry (CV) and Differential Pulse Voltammetry (DPV) of complex-1 in $\mathrm{CH}_{3} \mathrm{CN}$ using $\mathrm{TBAPF}_{6} 0.1 \mathrm{M}$ as electrolyte and $100 \mathrm{mV} / \mathrm{s}$ scan rate. Platinum wire was used as counter electrode and silver wire as the pseudoreference electrode. Ferrocene was added as internal standard. All potentials are reported versus $\mathrm{Ag} / \mathrm{AgCl}$.

\section{Conclusions and Outlook}

In summary, a rigid dinuclear $\operatorname{Ir}(\mathrm{III})$ complex showing high photoluminescence quantum yield (PLQY) in pure films was successfully used to fabricate LECs with and without ionic liquid additives. The devices show nearly instantaneous electroluminescence after biasing and electroluminescence quantum yield approaching $1 \%$. However, the lifetime of the devices was found to be limited, which we correlated with the irreversible oxidation of the complex as seen from electrochemical measurements. This work validates for the first time the use of highly luminescent dinuclear complexes in light-emitting electrochemical cells. Future studies will pursue materials with more efficient photoluminescence as well as improved electrochemical stability. 


\section{References}

[1] S.B. Meier, D. Tordera, A. Pertegás, C. Roldán-Carmona, E. Ortí, H.J. Bolink, Light-emitting electrochemical cells: recent progress and future prospects, Materials Today, 17 (2014) 217-223.

[2] A. Sandström, L. Edman, Towards High-Throughput Coating and Printing of Light-Emitting Electrochemical Cells: A Review and Cost Analysis of Current and Future Methods, Energy Technology, 3 (2015) 329-339.

[3] J.D. Slinker, J.A. DeFranco, M.J. Jaquith, W.R. Silveira, Y.-W. Zhong, J.M. Moran-Mirabal, H.G. Craighead, H.D. Abruña, J.A. Marohn, G.G. Malliaras, Direct measurement of the electricfield distribution in a light-emitting electrochemical cell, Nature Materials, 6 (2007) 894.

[4] P. Matyba, K. Maturova, M. Kemerink, N.D. Robinson, L. Edman, The dynamic organic $p-n$ junction, Nature Materials, 8 (2009) 672.

[5] S. van Reenen, P. Matyba, A. Dzwilewski, R.A.J. Janssen, L. Edman, M. Kemerink, A Unifying Model for the Operation of Light-Emitting Electrochemical Cells, Journal of the American Chemical Society, 132 (2010) 13776-13781.

[6] R.D. Costa, E. Ortí, H.J. Bolink, F. Monti, G. Accorsi, N. Armaroli, Luminescent lonic TransitionMetal Complexes for Light-Emitting Electrochemical Cells, Angewandte Chemie International Edition, 51 (2012) 8178-8211.

[7] A.F. Henwood, E. Zysman-Colman, Luminescent Iridium Complexes Used in Light-Emitting Electrochemical Cells (LEECs), Topics in Current Chemistry, 374 (2016) 36.

[8] S. Tang, A. Sandström, P. Lundberg, T. Lanz, C. Larsen, S. van Reenen, M. Kemerink, L. Edman, Design rules for light-emitting electrochemical cells delivering bright luminance at 27.5 percent external quantum efficiency, Nature Communications, 8 (2017) 1190.

[9] G. Li, D.G. Congrave, D. Zhu, Z. Su, M.R. Bryce, Recent advances in luminescent dinuclear iridium(III) complexes and their application in organic electroluminescent devices, Polyhedron, 140 (2018) 146-157.

[10] J. Fernández-Cestau, N. Giménez, E. Lalinde, P. Montaño, M.T. Moreno, S. Sánchez, M.D. Weber, R.D. Costa, Alkynyl bridged cyclometalated Ir2M2 clusters: impact of the heterometal in the photo- and electro-luminescence properties, Dalton Transactions, 45 (2016) 3251-3255.

[11] L.M. Cinninger, L.D. Bastatas, Y. Shen, B.J. Holliday, J.D. Slinker, Luminescent properties of a 3,5-diphenylpyrazole bridged Pt(ii) dimer, Dalton Transactions, (2019).

[12] R.D. Costa, G. Fernández, L. Sánchez, N. Martín, E. Ortí, H.J. Bolink, Dumbbell-Shaped Dinuclear Iridium Complexes and Their Application to Light-Emitting Electrochemical Cells, Chemistry - A European Journal, 16 (2010) 9855-9863.

[13] E.A. Plummer, J.W. Hofstraat, L. De Cola, Mono- and di-nuclear iridium(iii) complexes. Synthesis and photophysics, Dalton Transactions, (2003) 2080-2084.

[14] R.E. Daniels, S. Culham, M. Hunter, M.C. Durrant, M.R. Probert, W. Clegg, J.A.G. Williams, V.N. Kozhevnikov, When two are better than one: bright phosphorescence from nonstereogenic dinuclear iridium(iii) complexes, Dalton Transactions, 45 (2016) 6949-6962.

[15] S. Culham, P.-H. Lanoë, V.L. Whittle, M.C. Durrant, J.A.G. Williams, V.N. Kozhevnikov, Highly Luminescent Dinuclear Platinum(II) Complexes Incorporating Bis-Cyclometallating Pyrazine-Based Ligands: A Versatile Approach to Efficient Red Phosphors, Inorganic Chemistry, 52 (2013) 10992-11003.

[16] P.-H. Lanoë, C.M. Tong, R.W. Harrington, M.R. Probert, W. Clegg, J.A.G. Williams, V.N. Kozhevnikov, Ditopic bis-terdentate cyclometallating ligands and their highly luminescent dinuclear iridium(iii) complexes, Chemical Communications, 50 (2014) 6831-6834. 
[17] R.E. Daniels, L.K. McKenzie, J.R. Shewring, J.A. Weinstein, Valery N. Kozhevnikov, H.E. Bryant, Pyridazine-bridged cationic diiridium complexes as potential dual-mode bioimaging probes, RSC Advances, 8 (2018) 9670-9676.

[18] S.T. Parker, J.D. Slinker, M.S. Lowry, M.P. Cox, S. Bernhard, G.G. Malliaras, Improved Turnon Times of Iridium Electroluminescent Devices by Use of Ionic Liquids, Chem. Mater., 17 (2005) 3187-3190.

[19] Contact issues in electroluminescent devices from ruthenium complexes, Applied Physics Letters, 84 (2004) 807-809.

[20] M. Stössel, J. Staudigel, F. Steuber, J. Simmerer, A. Winnacker, Impact of the cathode metal work function on the performance of vacuum-deposited organic light emitting-devices, Applied Physics A, 68 (1999) 387-390.

[21] J. Huang, P.F. Miller, J.S. Wilson, A.J. de Mello, J.C. de Mello, D.D.C. Bradley, Investigation of the Effects of Doping and Post-Deposition Treatments on the Conductivity, Morphology, and Work Function of Poly(3,4-ethylenedioxythiophene)/Poly(styrene sulfonate) Films, Advanced Functional Materials, 15 (2005) 290-296. 